\title{
AIP Aponed phosics

Mid-infrared InAs0.79Sb0.21-based nBn photodetectors with Al0.9Ga0.2As0.1Sb0.9 barrier layers, and comparisons with InAs0.87Sb0.13 p-i-n diodes, both grown on GaAs using interfacial misfit arrays

A. P. Craig, A. R. J. Marshall, Z.-B. Tian, S. Krishna, and A. Krier

Citation: Applied Physics Letters 103, 253502 (2013); doi: 10.1063/1.4844615

View online: http://dx.doi.org/10.1063/1.4844615

View Table of Contents: http://scitation.aip.org/content/aip/journal/apl/103/25?ver=pdfcov

Published by the AIP Publishing

\section{Articles you may be interested in}

Direct minority carrier transport characterization of InAs/lnAsSb superlattice $\mathrm{nBn}$ photodetectors

Appl. Phys. Lett. 106, 071107 (2015); 10.1063/1.4913312

Room temperature performance of mid-wavelength infrared InAsSb nBn detectors

Appl. Phys. Lett. 105, 023512 (2014); 10.1063/1.4890465

Elimination of surface leakage in gate controlled type-II InAs/GaSb mid-infrared photodetectors Appl. Phys. Lett. 99, 183503 (2011); 10.1063/1.3658627

High-performance, long-wave $(\sim 10.2 \mu \mathrm{m})$ InGaAs/GaAs quantum dot infrared photodetector with quaternary In0.21Al0.21Ga0.58As capping

Appl. Phys. Lett. 99, 181102 (2011); 10.1063/1.3657142

Development of gold-doped $\mathrm{Hg} 0.79 \mathrm{Cd} 0.21 \mathrm{Te}$ for very-long-wavelength infrared detectors

Appl. Phys. Lett. 82, 4157 (2003); 10.1063/1.1581369

Frustrated by

old technology?

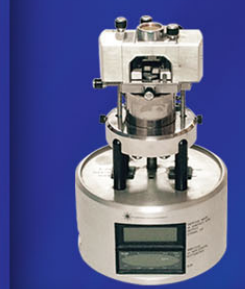

Is your AFM dead

and can't be repaired?

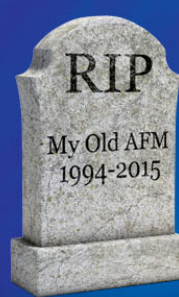

Sick of bad customer support?

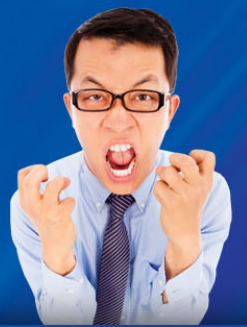

It is time to upgrade your AFM

Minimum $\$ 20,000$ trade-in discount for purchases before August 31st

Asylum Research is today's technology leader in AFM

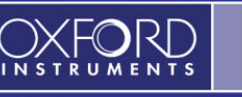




\title{
Mid-infrared InAs $0.79 \mathrm{Sb}_{0.21}$-based $n B n$ photodetectors with $\mathrm{Al}_{0.9} \mathrm{Ga}_{0.2} \mathrm{As}_{0.1} \mathrm{Sb}_{0.9}$ barrier layers, and comparisons with $\ln A s_{0.87} S_{0.13} p$-i-n diodes, both grown on GaAs using interfacial misfit arrays
}

\author{
A. P. Craig, ${ }^{1}$ A. R. J. Marshall, ${ }^{1}$ Z.-B. Tian, ${ }^{2}$ S. Krishna, ${ }^{2}$ and A. Krier $^{1}$ \\ ${ }^{1}$ Physics Department, Lancaster University, Lancaster LAI 4YB, United Kingdom \\ ${ }^{2}$ Center for High Technology Materials, Department of Electrical and Computer Engineering, \\ University of New Mexico, Albuquerque, New Mexico 87106, USA
}

(Received 19 September 2013; accepted 17 November 2013; published online 16 December 2013)

\begin{abstract}
InAs $_{0.79} \mathrm{Sb}_{0.21}$-based $n B n$ photodetectors were fabricated on a GaAs substrate, using the interfacial misfit array growth mode. Reductions in the dark current density of more than two orders of magnitude at $300 \mathrm{~K}$ and more than six orders of magnitude at $200 \mathrm{~K}$ were found, in comparison with an $\operatorname{InAs}_{0.87} \mathrm{Sb}_{0.13} p$ - $i$ - $n$ diode. At $-0.5 \mathrm{~V}$ applied bias, the dark currents were found to be diffusion limited above $150 \mathrm{~K}$. Background limited infrared photodetection and $R_{0} A$ values in excess of $10^{6} \Omega \mathrm{cm}^{2}$ were observed at $150 \mathrm{~K}$. Spectral response measurements revealed a cut-off wavelength of $5.3 \mu \mathrm{m}$ at $200 \mathrm{~K}$. (c) 2013 Author(s). All article content, except where otherwise noted, is licensed under a Creative Commons Attribution 3.0 Unported License. [http://dx.doi.org/10.1063/1.4844615]
\end{abstract}

Detectors for the mid-wavelength infrared (MWIR) spectral range $(3-5 \mu \mathrm{m})$ are of increasing technological interest for applications including defense, ${ }^{1}$ gas sensing, ${ }^{2}$ and thermal imaging. Currently, $p-n$ junction photodiodes made from InSb or $\mathrm{HgCdTe}$ are predominant. These devices typically require cooling to cryogenic temperatures to avoid excessive dark currents. The $n B n$, or $n$-barrier- $n$, photodetector is a unique design which strongly suppresses the dark currents and noise resulting from Shockley-Read-Hall (SRH) generation and surface states. ${ }^{3}$ Consisting of narrow-gap $n$ type absorption and contact layers separated by a wide gap barrier layer, the $n B n$ bandstructure prevents the flow of majority electrons, through a large conduction band offset, but allows the flow of minority holes, through a near-zero valence band offset. A small applied operating voltage falls almost completely across the barrier, separating the photogenerated carriers. The lack of significant electric field in the narrow-gap material prevents SRH generation and band-toband tunneling from taking place. In addition, the barrier layer is most often not etched in processing so that the shunt resistance for majority carriers associated with the surface states is very high. Consequently, $n B n$ photodetectors operating in the MWIR are known to have lower dark currents and noise than comparable $p-i-n$ photodiodes, allowing for reduced cooling requirements for background limited infrared photo-detection (BLIP) operation. ${ }^{3}$ The $n B n$ design has been modified by several authors ${ }^{4,5}$ to use an $\mathrm{InAs} / \mathrm{GaSb}$ strained-layer-superlattice (SLS) absorption region, with the flow of majority carriers blocked by a barrier of ternary AlGaSb. Reported cut-off wavelengths for these devices range from 4.3 to $5.2 \mu \mathrm{m}$, with specific detectivity, or $D^{*}$, values of around $1 \times 10^{9}$ Jones at $300 \mathrm{~K}$ and around 1 $\times 10^{11}$ Jones at $77 \mathrm{~K}$. Focal plane arrays (FPAs) have also been demonstrated, with dark currents equivalent to comparable $p-i-n$ devices with a passivation scheme. ${ }^{6}$ For midand long-wavelength detection, further alternatives, which are commercially available or under development, include quantum well infrared photodetectors (QWIPs) and quantum cascade detectors (QCDs). These also benefit from suppressed surface leakage currents and can be processed into photonic crystals, yielding significant performance benefits. QCDs with $D^{*}$ values of around $1 \times 10^{11}$ Jones at $108 \mathrm{~K}$ have been reported, for devices with a $3.96 \mu \mathrm{m}$ cut-off wavelength. ${ }^{7}$ In this work, an Interfacial Misfit (IMF) array was used to grow $n B n$ detectors with bulk $\operatorname{InAs}_{0.79} \mathrm{Sb}_{0.21}$ absorption layers on a GaAs substrate. Advantages of this approach include lower cost, when compared with native substrates such as GaSb and InAs, as well as optical transparency at infrared wavelengths - a significant advantage in flip-chip mounting for FPAs, where incident radiation is directed through the substrate. The IMF growth mode allows material of good crystalline quality to be deposited, "buffer-free," onto lattice-mismatched substrates. ${ }^{8}$ Herein, the strain is relieved within several monolayers of the interface by a periodic, self-ordered network consisting purely of $90^{\circ}$ edge dislocations. These propagate laterally, rather than into the overlying epilayer, and defect densities as low as 5 $\times 10^{5} \mathrm{~cm}^{-2}$ have been reported for bulk GaSb on GaAs. ${ }^{9}$ Derivative devices have already been demonstrated, including lasers, ${ }^{10}$ light emitting diodes (LEDs), ${ }^{11}$ and photodiodes, ${ }^{12}$ and have shown similar levels of performance to their lattice-matched equivalents. In addition, SLS-based $n B n$ detectors have already been demonstrated using the IMF growth mode. ${ }^{13}$ The suppression of the SRH generation profile by the $n B n$ design is particularly suited to devices based on mismatched growth since traps at any remaining threading dislocations will contribute little to the dark currents. Indeed, in this work, the InAs $\mathrm{As}_{0.79} \mathrm{Sb}_{0.21}$ absorption layer was grown with a mismatched composition on a GaSb buffer layer, allowing for extended wavelength detection, yet diffusion limited dark currents were still demonstrated. The $n B n$ barrier layer composition was also modified to include a small Ga mole fraction. This suppresses oxidation of the exposed surface, which is a significant challenge in 
processing, avoiding potential issues with long term reliability. An InAs ${ }_{0.87} \mathrm{Sb}_{0.13} p-i-n$ structure, lattice matched to $\mathrm{GaSb}$, was also grown on a GaAs substrate using an IMF. This provided a benchmark against which to compare the $n B n$ structure, with the minimum possible threading dislocation density under the IMF growth mode.

Growth was performed using a VG-V80H MBE reactor, with thermal effusion K-cells providing In, $\mathrm{Ga}$, and $\mathrm{Al}$ fluxes and valved cracker cells providing $\mathrm{As}_{2}$ and $\mathrm{Sb}_{2}$ fluxes. In situ reflection high energy electron diffraction (RHEED) was used to calibrate the growth rates, by using a photomultiplier tube to monitor the intensity oscillations, and to monitor surface reconstruction. Oxide desorption from the GaAs substrate was performed first, at $600^{\circ} \mathrm{C}$. The growth temperature was subsequently reduced to $570^{\circ} \mathrm{C}$ for the growth of the GaAs buffer layer. The IMF interface was then initiated, through a brief pause with no applied $\mathrm{As}_{2}$ flux followed by the application of $\mathrm{Sb}_{2}$ flux. At the same time, the temperature was reduced to $510^{\circ} \mathrm{C}$ for the growth of the $\mathrm{GaSb}$ buffer layer, which was used to transform the lattice constant to $6.1 \AA$. The InAs ${ }_{0.79} \mathrm{Sb}_{0.21}$ absorption layer was grown at $450{ }^{\circ} \mathrm{C}$ and $0.9 \mathrm{MLs}^{-1}$. The quaternary barrier layer was grown at $490{ }^{\circ} \mathrm{C}$ and $0.8 \mathrm{MLs}^{-1}$. The $\operatorname{InAs}_{0.87} \mathrm{Sb}_{0.13}$ $p-i-n$ diode was grown under the same conditions as the $n B n$. The $n$-type dopant was GaTe and the $p$-type dopant Be. The $p-i-n$ has an unintentionally doped intrinsic region, with a thickness of $1.1 \mu \mathrm{m}$. In processing, annular top contacts were evaporated first, using $\mathrm{Ti} / \mathrm{Au}$, and circular mesas were then defined using $\mathrm{C}_{6} \mathrm{H}_{8} \mathrm{O}_{7}: \mathrm{H}_{2} \mathrm{O}_{2}(2: 1)$. The lower contacts were positioned well away from the mesas and were evaporated, also using $\mathrm{Ti} / \mathrm{Au}$, after etching away the barrier layer and into the contact layer, using $\mathrm{HCl}: \mathrm{H}_{2} \mathrm{O}_{2}: \mathrm{H}_{2} \mathrm{O}(1: 1: 5)$ and $\mathrm{C}_{6} \mathrm{H}_{8} \mathrm{O}_{7}: \mathrm{H}_{2} \mathrm{O}_{2}(2: 1) . \mathrm{H}_{3} \mathrm{PO}_{4}: \mathrm{H}_{2} \mathrm{O}_{2}: \mathrm{H}_{2} \mathrm{O}(1: 1: 10)$ was used to etch the $p-i-n$ diode. After fabrication, $\mathrm{x}$-ray diffraction (XRD) and temperature dependent spectral response and current density $(J-V)$ measurements were made. Devices of diameters $200 \mu \mathrm{m}, 400 \mu \mathrm{m}$, and $800 \mu \mathrm{m}$ were bonded to a TO-05 header and tested concurrently in an Oxford Instruments cryostat, using multiple Keithley 2400 SourceMeters. The header was capped to shield the $300 \mathrm{~K}$ background. $400 \mu \mathrm{m}$ diameter devices were also measured in a Lakeshore TTPX low-temperature Probe Station, equipped with an optional radiation shield. Spectral response was measured using a Nicolet 6700 Fourier Transform Infrared (FTIR) Spectrometer. X-ray diffraction results were obtained using a Bede QC200 Diffractometer. Quantum efficiency (QE) was measured at $300 \mathrm{~K}$ using a calibrated $1.55 \mu \mathrm{m}$ laser source, incident on the device under test via a cleaved, single mode optical fiber.

Details of the $n B n$ detector structure are given in Figure 1(a). The compositions were found by XRD, as shown in Figure 2(a). XRD for the $p-i-n$ structure is shown in Figure 2(b). Fittings were performed using RADS Mercury software. The barrier layer of the $n B n$ was assumed to be pseudomorphic; this is supported by a critical thickness calculation for strained layer growth of $66 \mathrm{~nm}$, using the method of People and Bean. ${ }^{14}$ The details of the bandstructure were then calculated, using the strain dependent model of Krijn, ${ }^{15}$ giving the valence band offset as approximately $28 \mathrm{meV}$, in a type-II alignment, illustrated in Figure 1(b). Spectral response

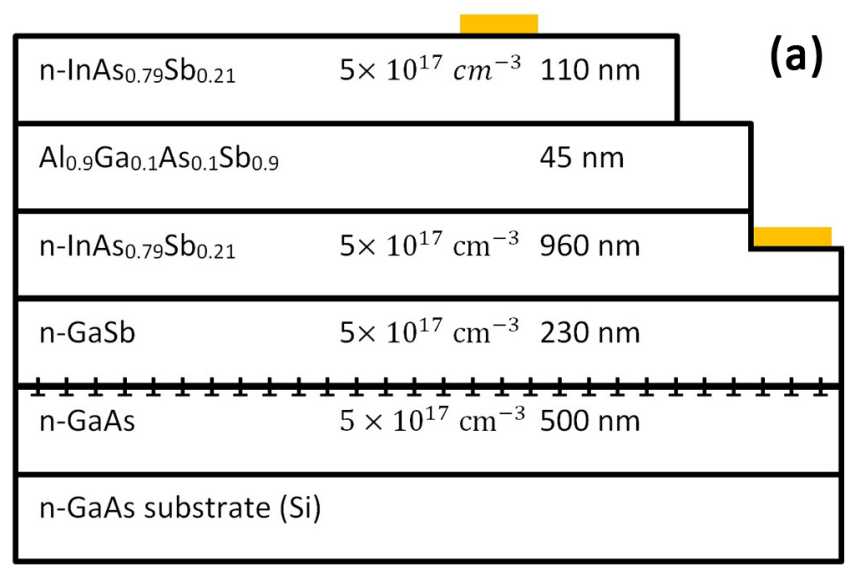

$\pm \perp \perp \perp \pm \mathrm{IMF}$

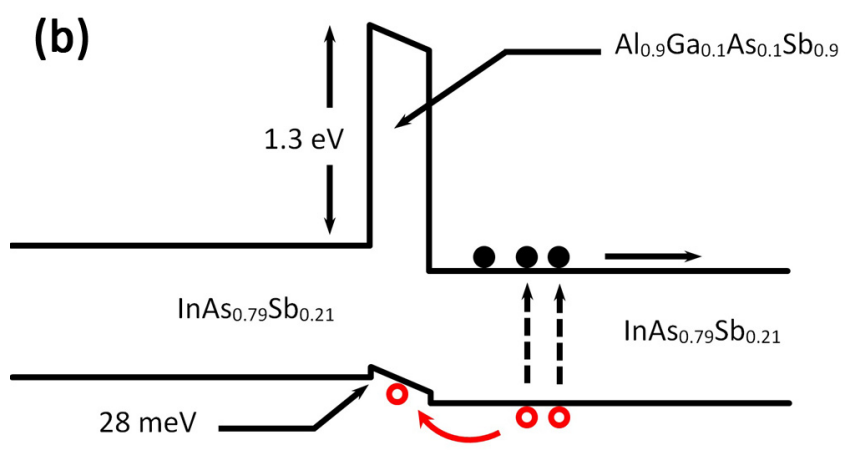

FIG. 1. (a) Layer thicknesses and compositions for the $n B n$ structure, found from RHEED calibration and RADS Mercury XRD simulation, assuming a strained barrier layer. (b) The results of the bandstructure calculation. The $n B n$ design allows the flow of minority holes whilst preventing the flow of majority electrons at the barrier, which separates the photogenerated carriers.

curves for the $n B n$ detector are shown in Figure 3, indicating a cut-off wavelength of around $4.93 \mu \mathrm{m}$ at $60 \mathrm{~K}$, which is comparable to binary InSb, effectively covering the MWIR atmospheric transmission window. By plotting the square of the photoresponse against energy, the absorption layer bandgap was found to be $0.27 \mathrm{eV}$, indicating a composition in close agreement with the XRD fitting. Strain at the $\mathrm{GaSb} / \mathrm{InAsSb}$ interface, residual impurities or the Franz-Keldysh effect could be responsible for the tails seen in the 5-5.5 $\mu \mathrm{m}$ region of the spectral response. In Figure 4, Arrhenius plots of the dark current density are compared with results from the InAs $\mathrm{As}_{0.87} \mathrm{Sb}_{0.13} p-i$ - $n$. Close agreement was observed between the $n B n$ current densities for the three device areas, showing the suppression of surface currents and confirming the reliability of the data. At $-0.1 \mathrm{~V}$ applied bias, the dark current densities of the $p-i-n$ are more than two orders of magnitude greater than the $n B n$ at $300 \mathrm{~K}$ and more than six orders greater at $200 \mathrm{~K} . R_{0} A$ values for the $p-i-n$ were measured to be approximately $3 \times 10^{-2} \Omega \mathrm{cm}^{2}$ at $220 \mathrm{~K}$. While these are significantly lower than values for $\mathrm{InAs}_{0.89} \mathrm{Sb}_{0.11} p-i-n$ diodes grown on native $\mathrm{GaSb}$ substrates, reported to be approximately $1 \Omega \mathrm{cm}^{2}$ at the same temperature, ${ }^{16} R_{0} A$ values for the $n B n$ were found to be in excess of $3 \times 10^{3} \Omega \mathrm{cm}^{2}$ at $220 \mathrm{~K}$ and in excess of $10^{6} \Omega \mathrm{cm}^{2}$ at $150 \mathrm{~K}$. In comparison, typical InSb detectors require cooling to roughly $90 \mathrm{~K}$ to achieve $R_{0} A$ values of $10^{6} \Omega \mathrm{cm}^{2} .{ }^{17}$ Notably, these improvements are observed in spite of the mismatched 

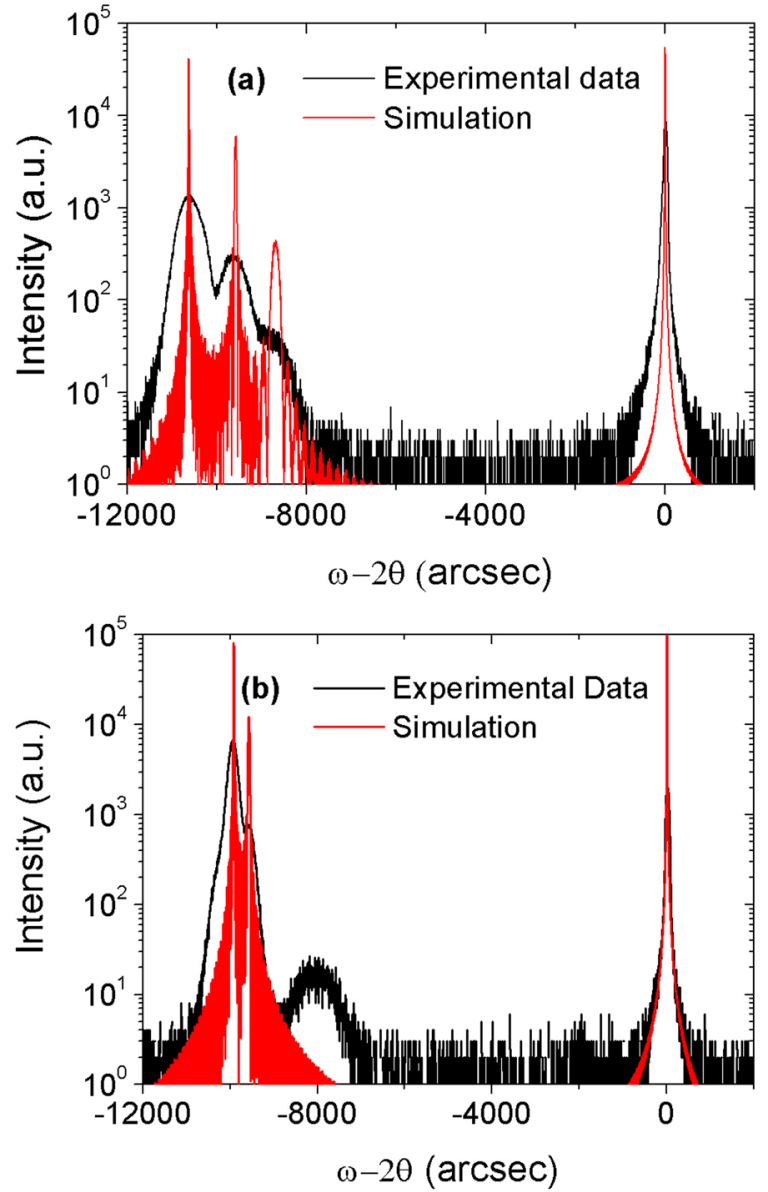

FIG. 2. XRD data and fitted RADS Mercury simulation giving compositions for (a) the $n B n$ structure, under the assumption that the barrier layer is pseudomorphic, and (b) the $\mathrm{InAs}_{0.87} \mathrm{Sb}_{0.13} p-i-n$ diode.

substrate and an additional lattice-mismatch in the absorption layer, showing that the SRH process is strongly suppressed in the $n B n$ whilst, at the same time, achieving an extended cut-off wavelength. At $-0.5 \mathrm{~V}$ applied bias, unshielded measurements revealed a BLIP temperature of approximately $150 \mathrm{~K}$. The dashed line shows a fitting to the form of the diffusion current, $J_{\text {diff, }}$, limited regime, known from the relationship between the square of the intrinsic carrier concentration, $n_{i}^{2}$, and the bandgap, $E_{g}$,

$$
J_{\text {diff }} \propto n_{i}^{2} \propto T^{3} \exp \left(\frac{-E_{g}}{k T}\right),
$$

where $k$ is the Boltzmann constant and the density of states contributes the factor of $T^{3}$. An activation energy of $0.29 \mathrm{eV}$ was found, which is close to the bandgap of the absorption layer, corroborating the suppression of the SRH process above approximately $150 \mathrm{~K}$. Below $150 \mathrm{~K}$, the dark currents are generally limited by the noise floor of the measurement setup, though a tunneling mechanism could also be contributing to the data measured for $-0.5 \mathrm{~V}$ bias. Gradients in this region were too small to be linked with SRH recombination, and surface currents are known to be blocked by the barrier. In contrast, for the $\operatorname{InAs}_{0.87} \mathrm{Sb}_{0.13} p-i-n$ diode, the gradient is consistent with SRH limited dark currents, as shown in the inset of Figure 4. The QE was measured to be $22 \%$ although it is noted that the large absorption coefficient of the contact

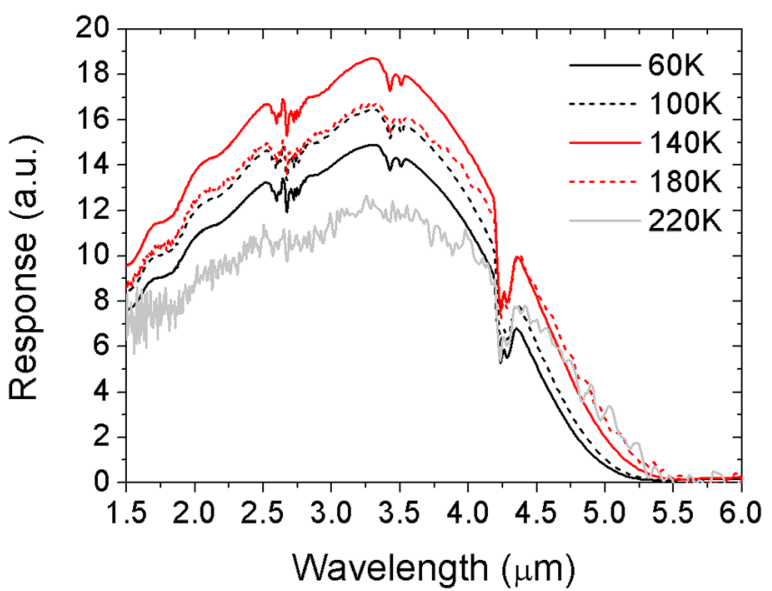

FIG. 3. Spectral response curves for the $n B n$ detector, between 60 and $220 \mathrm{~K}$, taken using a Nicolet 6700 FTIR. Extrapolation of the squared responsivity, plotted against energy, was used to determine the bandgap.

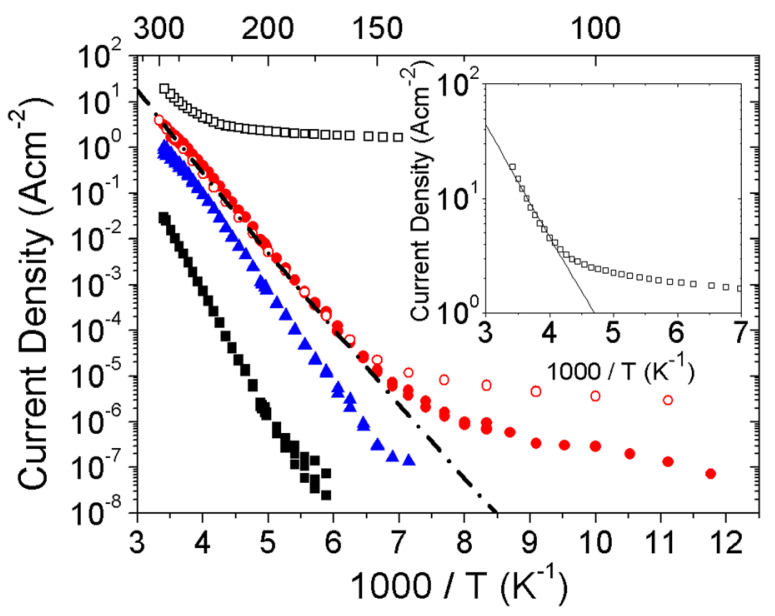

FIG. 4. Arrhenius plots, with shielded $300 \mathrm{~K}$ background, at $-0.1 \mathrm{~V}$ (ם), $-0.3 \mathrm{~V}(\boldsymbol{\Delta})$, and $-0.5 \mathrm{~V}(\boldsymbol{\bullet})$, and the $\operatorname{InAs}_{0.87} \mathrm{Sb}_{0.13} \mathrm{p}-\mathrm{i}-\mathrm{n}(\square)$, at $-0.1 \mathrm{~V}$. The unshielded data at $-0.5 \mathrm{~V}(\mathrm{O})$ reveals a BLIP temperature of roughly $150 \mathrm{~K}$. The dashed line shows the fitting to the diffusion current limited regime. The inset shows the data for the $p-i-n$ compared with the gradient expected for SRH limited dark currents.

layer at $1.55 \mu \mathrm{m}$ and lack of an anti-reflective coating limit this figure to a degree. The diffusion length for holes was estimated at $300 \mathrm{~K}$, using an approach similar to the area-dependent method of Plis, ${ }^{18}$ and found to be $2.6 \mu \mathrm{m}$.

In summary, $n B n$ detectors were grown on a GaAs substrate using an IMF Array. InAs ${ }_{0.79} \mathrm{Sb}_{0.21}$ absorption layers allowed for extended wavelength detection while quaternary $\mathrm{Al}_{0.9} \mathrm{Ga}_{0.2} \mathrm{As}_{0.1} \mathrm{Sb}_{0.9}$ barrier layers allowed for a suppression of surface oxidation. Devices were compared with an $\mathrm{InAs}_{0.87} \mathrm{Sb}_{0.13} p$-i-n diode, also grown on a GaAs substrate using an IMF Array. At $200 \mathrm{~K}$, reductions in the dark current density greater than six orders of magnitude were observed. Significantly, these devices could be used for large area, low cost focal plane arrays, supporting high operating temperatures.

The authors wish to thank the UK Engineering and Physical Sciences Research Council for the studentship provided to A. P. Craig (Grant No. EP/P505585/1) and the Royal Academy of Engineering for the fellowship awarded to A. R. J. Marshall (Grant No. EP/H043993/1). 
${ }^{1}$ I. M. Baker, S. S. Duncan, and J. W. Copley, Proc. SPIE 5406, 133 (2004).

${ }^{2}$ A. Krier and W. Suleiman, Semicond. Sci. Technol. 13, 950 (1998).

${ }^{3}$ S. Maimon and G. W. Wicks, Appl. Phys. Lett. 89, 151109 (2006).

${ }^{4}$ G. Bishop, E. Plis, J. B. Rodriguez, Y. D. Sharma, L. R. Dawson, and S. Krishna, J. Vac. Sci. Technol. B 26(3), 1145 (2008).

${ }^{5}$ J. B. Rodriguez, E. Plis, G. Bishop, Y. D. Sharma, H. Kim, L. R. Dawson, and S. Krishna, Appl. Phys. Lett. 91, 043514 (2007).

${ }^{6}$ H. S. Kim, E. Plis, J. B. Rodriguez, G. D. Bishop, Y. D. Sharma, L. R. Dawson, S. Krishna, J. Bundas, R. Cook, D. Burrows, R. Dennis, K. Patnaude, A. Reisinger, and M. Sundaram, Appl. Phys. Lett. 92, 183502 (2008).

${ }^{7}$ F. R. Giorgetta, E. Baumann, M. Graf, Q. Yang, C. Manz, K. Köhler, H. E. Beere, D. A. Ritchie, E. Linfield, A. G. Davies, Y. Fedoryshyn, H. Jäckel, M. Fischer, J. Faist, and D. Hofstetter, IEEE J. Quantum Electron. 45, 1039 (2009).

${ }^{8}$ S. H. Huang, G. Balakrishnan, A. Khoshakhlagh, A. Jallipalli, L. R. Dawson, and D. L. Huffaker, Appl. Phys. Lett. 88, 131911 (2006).

${ }^{9}$ S. Huang, G. Balakrishnan, and D. L. Huffaker, J. Appl. Phys. 105, 103104 (2009).
${ }^{10}$ J. B. Rodriguez, L. Cerutti, and E. Tournié, Appl. Phys. Lett. 94, 023506 (2009).

${ }^{11}$ M. Mehta, G. Balakrishnan, S. Huang, A. Khoshakhlagh, A. Jallipalli, P. Patel, M. N. Kutty, L. R. Dawson, and D. L. Huffaker, Appl. Phys. Lett. 89, 211110 (2006).

${ }^{12}$ K. C. Nunna, S. L. Tan, C. J. Reyner, A. R. J. Marshall, B. Liang, A. Jallipalli, J. P. R. David, and D. L. Huffaker, IEEE Photonics Technol. Lett. 24, 218 (2012).

${ }^{13}$ E. Plis, J. B. Rodriguez, G. Balakrishnan, Y. D. Sharma, H. S. Kim, T. Rotter, and S. Krishna, Semicond. Sci. Technol. 25, 085010 (2010).

${ }^{14}$ R. People and J. C. Bean, Appl. Phys. Lett. 47(3), 322 (1985).

${ }^{15}$ M. P. C. M. Krijn, Semicond. Sci. Technol. 6, 27 (1991).

${ }^{16}$ A. Rogalski, R. Ciupa, and W. Larkowski, Solid-State Electron. 39, 1593 (1996).

${ }^{17}$ A. Rogalski, Infrared Detectors, 2nd ed. (CRC Press, Boca Raton, FL, 2011).

${ }^{18}$ E. A. Plis, S. Myers, M. N. Cutty, J. Malifert, E. P. Smith, S. Johnson, and S. Krishna, Proc. SPIE 7945, 79451R-1 (2011). 